\title{
Comportement hydrique au cours de la saison sèche et place dans la succession de trois arbres guyanais: Trema micrantha, Goupia glabra et Eperua grandiflora
}

\author{
DY Alexandre *
}

ORSTOM, Cayenne, Guyane

(Reçu le 8 janvier 1990; accepté le 19 mai 1990)

\begin{abstract}
Résumé - Le potentiel hydrique de base, le potentiel hydrique minimal et la conductance stomatique maximale ont été mesurés sur 2 arbres pionniers (Trema micrantha, Goupia glabra) et un arbre forestier (Eperua grandiflora) au cours des 2 périodes sèches de 1987, à Cayenne, Guyane française. Tréma est localement spontané tandis que les goupis proviennent de sauvageons transplantés et les wapas de semis. Les plants des 3 espèces ont environ 2 ans et demi, puis 3 ans au moment des mesures. Les résultats montrent des différences de comportement importantes entre les 3 espèces. Tréma maintient un potentiel hydrique et une transpiration élevés (mais perd une partie de sa surface foliaire) tandis que le goupi accuse une forte baisse de potentiel et doit réguler sa transpiration. Le comportement du wapa est intermédiaire. Les mesures de laboratoire ont montré que la teneur en matière sèche des feuilles, la teneur en eau à perte de turgescence, la teneur en eau sublétale et celle de fanaison augmentent du tréma au goupi puis au wapa. Le potentiel osmotique est plus bas chez le wapa que chez les 2 autres espèces et la transpiration plus rapide chez le tréma que chez les 2 autres. Ces différences sont en grande partie liées à la morphologie racinaire des 3 espèces.
\end{abstract}

Trema micrantha / Goupia glabra / Eperua grandiflora / Guyane française / potentiel hydrique / conductance stomatique

Summary - Water characteristics and successional position of three tree species in French Guyana in the dry season: Trema micrantha, Goupia glabra, Eperua grandiflora. Predawn water potential, minimum water potential and maximum stomatal conductance were measured in 2 pioneer species (Trema micrantha and Goupia glabra) and a forest tree (Eperua grandiflora) during the 2 dry spells in 1987 at Cayenne. 1987 was a very dry year but cannot be considered as exceptional. Trema growh was spontaneous, Goupia was grown from transplanted wild seedlings and Eperua was directly sown. All were about 3-y-old.

Results show marked differences between the 2 pioneer species. Trema maintains a high water potential and transpiration (but a decreased foliar area) while Goupia endures a low water potential and closes its stomata. Eperua appears intermediate. Laboratory measurements show a dry matter content, a turgescence lost point, a sublethal RWC and a wilting RWC increasing from Trema to Goupia and from Goupia to Eperua. Osmotic potential is lower for Eperua and transpiration speed higher for Trema than for the other 2 species respectively. All in all there is a general behavioural

\footnotetext{
* Adresse actuelle : ORSTOM, Ouagadougou, BP 182, Ouagadougou, Burkina-Faso
} 
gradient from a large water expenditure in the early successional species to a tighter water control in the late successional species. The main reason for these differences may be found in the root morphologies.

\section{tropical forest / water potential / stomatal conductance / French Guyana / Trema micrantha / Goupia glabra / Eperua grandiflora}

\section{INTRODUCTION}

La Guyane française appartient à la zone climatique de la forêt dense humide subéquatoriale qui pourrait se définir par l'absence de contraintes hydriques majeures pour la végétation. Ainsi, à Cayenne, la pluviométrie moyenne annuelle est proche de $3000 \mathrm{~mm}$, soit deux fois supérieure à la demande évaporatrice. A priori, le facteur hydrique n'est donc pas celui qui détermine le plus la végétation, si ce n'est par son excès.

Globalement ce point de vue est peu contestable; en revanche, la variabilité tant inter- que intra-annuelle de la pluviosité (Alexandre, 1989) et les conditions pédologiques ou biologiques des stations peuvent conférer une grande importance aux petites différences de comportement de chaque espèce. C'est sur des plantes jeunes, plus fragiles, moins bien enracinées et en milieu ouvert que la contrainte hydrique peut se révéler importante et un facteur de différenciation de la végétation.

\section{MATÉRIEL ET MÉTHODE}

Les mesures ont été conduites à Cayenne, sur un site en bordure de la réserve forestière du Montabo, sur 2 espèces pionnières bien caractéristiques: Trema micrantha et Goupia glabra (le goupi). Ces deux arbres présentent l'avantage, sur le plan de l'expérimentation écophysiologique, de posséder une architecture identique (modèle de Roux, Hallé et Oldeman, 1970) et des feuilles de taille et de disposition semblables.
Les plants de Trema micrantha sont spontanés et datent de fin 1985, époque à laquelle de jeunes plants de Goupia glabra, prélevés en forêt, piste de Saint-Elie, ont été repiqués. Les tréma atteignent une hauteur de $8 \mathrm{~m}$ pour une circonférence de $28,5 \mathrm{~cm}$ à $1,30 \mathrm{~m}$. Les goupi, au nombre de 16 , atteignent $3,65 \mathrm{~m}$ (moyenne $2,78 \mathrm{~m}$ ) pour un diamètre de $18,3 \mathrm{~cm}$ (moyenne $10,0 \mathrm{~cm})$.

Des mesures complémentaires ont été effectuées sur d'autres espèces héliophiles également spontanées, présentes sur place (Cecropia palmata, Didymopanax morototoni, Solanum surinamense...). Toutes ces mesures ont apporté des résultats très proches de ceux obtenus avec Trema micrantha.

Quelques mesures ont également été conduites sur Eperua grandiflora (ou wapa). II s'agissait de brins issus d'un semis sur place en janvier 1985. Le wapa est un grand arbre forestier dont l'écologie a fait l'objet de travaux récents (Forget, 1988). La petite taille $(40 \mathrm{~cm}$ de haut) des quelques brins survivants de cette espèce, ici bien en dehors de son habitat normal, explique le petit nombre de mesures effectuées, malgré l'intérêt qu'elles auraient pu présenter.

II faut noter que Goupia glabra est totalement absent de l'lle de Cayenne et de la réserve forestière du Montabo en particulier, mais devient très abondant à sa périphérie. Nous en avons ainsi rencontré et étudié d'importantes populations dans le périmètre ONF de Macouria ainsi que Chemin Mogès à Stoupan, à environ $15 \mathrm{~km}$ de Cayenne.

Chez le goupi, comme chez toute espèce plastique (Bannister, 1971), les caractères écophysiologiques peuvent différer de façon sensible selon le site de croissance. Sur la piste de Saint-Élie nous avons observé des feuilles de goupi plus grandes, plus épaisses, plus riches en matière sèche et avec une conductance stomatique maximale plus élevée qu'à Cayenne. D'après les mesures de Colin (Colin, 1989 in Lacoste, 1990), ce dernier caractère peut être rap- 
porté à l'humidité atmosphérique élevée en forêt. II importe donc de souligner que tous les paramètres rapportés ci-dessous proviennent du site de Cayenne.

Les mesures effectuées ont porté sur les paramètres habituels. In situ, nous avons mesuré le potentiel de base, le potentiel minimal et la conductance maximale. Ces mesures ont été complétées au laboratoire par des expériences conduisant à la courbe pression-volume, au déficit sublétal ou à la cinétique de transpiration de rameaux coupés.

\section{Mesures de potentiel}

Le potentiel hydrique de base (Aussenac, 1985) et le potentiel minimal ont été mesurés à l'aide d'une chambre à pression PMS (PMS Instrument Co, Corvallis, OR, USA). Quand les stomates sont fermés, la mesure ne pose pas de problèmes (potentiel de base ou potentiel minimal de plantes stressées). Quand les plantes transpirent activement, le potentiel s'abaisse très rapidement au cours de la mesure jusqu'à la fermeture hydroactive des stomates. Afin d'obtenir une estimation du potentiel in vivo, on a effectué les mesures de façon très rapide et chronométrée sur des couples de rameaux. En corrélant la différence de potentiel observée avec la différence de durée de mesure, on obtient un facteur de correction qui permet d'estimer le potentiel in vivo.

Sur goupi et tréma, nous avons effectué les mesures sur des extrémités de rameaux comportant 3 ou 4 feuilles. Pour Eperua grandiflora, on a utilisé la partie terminale de la feuille avec 2 paires de folioles.

Les mesures de potentiel minimal ont été effectuées vers $13 \mathrm{~h}$, heure locale (= heure solaire).

\section{Conductances stomatiques maximales}

Les mesures de conductances stomatiques ont été effectuées à l'aide du poromètre à diffusion MK3 de Delta-T Devices (Cambridge, UK). Compte tenu de l'humidité ambiante, la gamme de mesure utilisée est celle qui fait passer l'humidité relative de la chambre de 0,65 à 0,75 .
Les mesures sont effectuées sur la $4^{e}$ feuille en partant de l'extrémité des rameaux. En période de croissance, c'est souvent la feuille de ce rang qui montre la conductance la plus élevée et la variabilité la plus faible. La figure 1 illustre ce fait à partir d'une série de mesures sur le goupi. Le tréma réagit comme le goupi mais possède moins de vieilles feuilles; chez le wapa, les jeunes feuilles, colorées en rouge, ont une conductance proche de 0 . Cette résistance élevée des jeunes feuilles irrégulière pour les plus vieilles, a été observée chez d'autres espèces tropicales par Reich et Borchert (1988). Sur chaque plante on mesure la conductivité de 4 feuilles situées aux 4 points cardinaux, à mihauteur du feuillage. Compte tenu de la variabilité des mesures et de la précision possible, on a mesuré la conductivité sur 4 individus pour le goupi, 3 pour le tréma et 2 pour Eperua grandiflora. Tous les arbres rencontrés ont des feuilles hypostomatiques, aussi les mesures de conductance ont-elles été limitées à la face abaxiale.

Moyennant un équilibrage thermique suffisant, un entretien soigneux de la propreté de la cuvette el un étalonnage à chaque série de mesures, la précision finale est de l'ordre de $5 \%$ pour les conductances moyennes, allant de 0,1 à $1 \mathrm{~cm} / \mathrm{s}$, ce qui est tout à fait satisfaisant. Pour les valeurs très élevées ou très faibles de

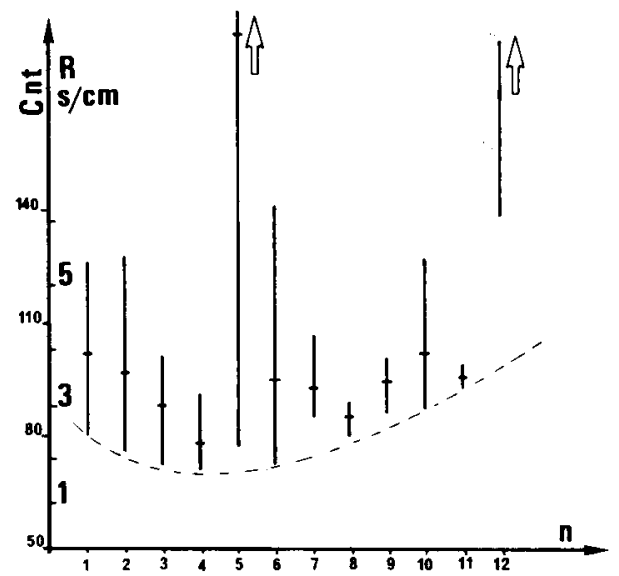

Fig 1. Distribution de la résistance stomatique en fonction du rang de la feuille à partir de l'extrémité du rameau. Exemple obtenu à partir de Goupia glabra, mai 1985. Le trait vertical indique l'intervalle de confiance. 
conductance, pour lesquelles les problèmes techniques ne sont pas résolus, l'incertitude est de l'ordre de $25 \%$.

En saison sèche, chez un grand nombre d'espèces, la conductance augmente rapidement le matin puis diminue progressivement au cours de la journée (résultat non publié). Afin d'estimer la conductance maximale, les mesures ont donc été effectuées, dans la mesure du posible, en milieu de matinée.

\section{Autres mesures}

Les courbes pression-volume, les déficits sublétaux et les mesures de transpiration de rameaux coupés ont été obtenus avec une balance Mettler électronique, sensible au mg. $\mathrm{Ce}$ matériel permet un gain de temps inestimable, mais ne supporte pas les variations rapides d'humidité relative (HR) et de température du terrain. Toutes les pesées ont donc été effectuées au laboratoire. Les échantillons ont été saturés à l'obscurité et à $7^{\circ} \mathrm{C}$, puis séchés à $80^{\circ} \mathrm{C}$. Pour le déficit sublétal (DSL), les temps de ressaturation adoptés après essais sont de 6 et $24 \mathrm{~h}$. Pour les courbes pression-volume, nous avons adopté un protocole discontinu, avec pesée du rameau + joint à chaque pression d'équilibre obtenue dans la chambre PMS. Les transpirations de rameau coupé ont été suivies à l'intérieur d'une enceinte ventilée de notre fabrication (éclairement de $250-350 \mathrm{~W} / \mathrm{m}^{2}$ obtenu par lampe à iode, lampe à mercure et filtre à eau; température $28^{\circ} \mathrm{C}$ et HR $60 \%$ contrôlées par climatiseur et déshumidifieur). Les courbes pression-volume, celles de transpiration et les diagrammes de ressaturation ont été exploités graphiquement.

Le tréma et le goupi ne possèdent pas de gomme ou de résine susceptibles de boucher les vaisseaux et de gêner la ressaturation du matériel. En revanche, chez le wapa, ce genre de problème peut se produire comme en témoignent quelques apparentes sursaturations avec du matériel blessé. Les résultats concernant le wapa sont à interpréter avec prudence.

La pluviométrie est mesurée avec un pluviomètre standard relevé quotidiennement à $18 \mathrm{~h}$.

L'indice foliaire de tréma a été estimé par point-contact avec une perche verticale en profitant du port fortement planophile de l'arbre.

\section{RÉSULTATS}

L'année 1987 a été particulièrement sèche, avec un "petit été de mars" très accusé. La faible pluviosité a des répercussions sur la végétation comme l'indiquent les figures 2 et 3 .

Le comportement du tréma et celui du goupi sont bien différents. Tréma conserve un potentiel de base proche de 0 et une transpiration élevée contrairement au
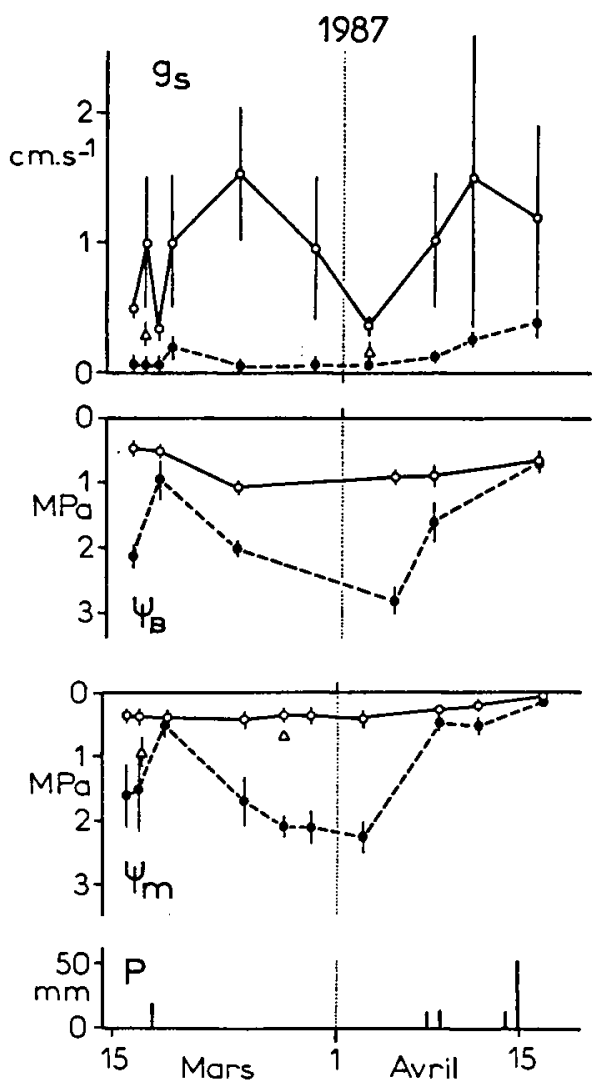

Fig 2. Pluviométrie $(p)$, potentiel minimal $\left(\Psi_{m}\right)$, potentiel de base $\left(\psi_{B}\right)$, et conductance maximale (gs) de Trema micrantha (- - - -), Goupia glabra (-O-) et Eperua grandiflora $(\Delta)$ au cours du "petit été de mars" en 1977, à Cayenne. Le trait vertical indique l'intervalle de confiance. 

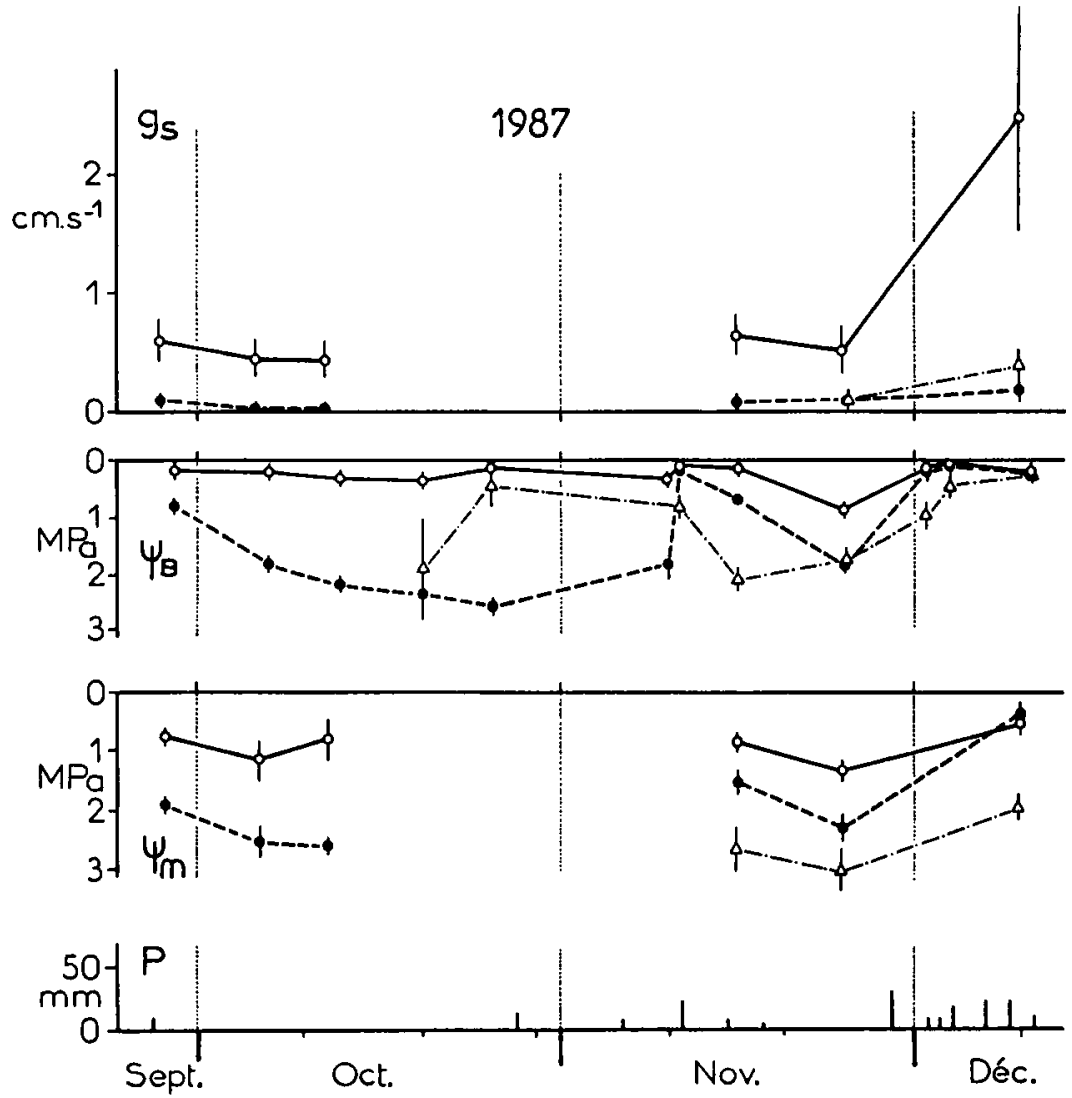

Fig 3. Pluviométrie $(p)$, potentiel minimal $\left(\psi_{m}\right)$, potentiel de base $\left(\psi_{B}\right)$ et conductance maximale (Gs) de Trema micrantha (- - - ), Goupia glabra (-O-) et Eperua grandiflora $(-\cdot-\Delta-\cdot)$ ) au cours de la saison sèche de 1977, à Cayenne. Le trait vertical indique l'intervalle de confiance.

goupi qui voit son potentiel dimuner fortement et qui finit par fermer complètement ses stomates. Le comportement du wapa semble intermédiaire entre celui du tréma et du goupi, ce qui est paradoxal si l'on considère que le goupi est situé entre le tréma, espèce très héliophile, et le wapa, sciaphile. La différence de comportement entre le goupi et le tréma est visible lors des 2 épisodes secs de l'année 1987. Cependant des différences apparaissent quant à la reprise d'activité normale au retour des pluies. Lors du petit été de mars, où la sécheresse du milieu n'est que rela- tive, la reprise de la transpiration ou la remontée des potentiels sont très rapides, particulièrement dans le cas du tréma. Au contraire, au cours de la saison sèche d'août à décembre, le déficit hydrique est considérable et les arbres ne reprennent que lentement leur pleine activité transpiratoire. En fin de saison sèche, on constate que le potentiel du wapa descend audessous de celui du goupi et que sa reprise est particulièrement lente. La plante semble touchée dans ses mécanismes internes, alors que lors de l'épisode précédent, elle avait émis, en pleine période 
sèche, des feuilles neuves, traduisant ainsi un bon état de santé. Les tests de laboratoire confirment cette différence de sensibilité à la sécheresse.

Considérons tout d'abord les teneurs en matière sèche des feuilles telles qu'on peut les calculer à partir des nombreux échantillons prélevés. Elles sont en moyenne de $31 \pm 0,5$ chez tréma, $33,1 \pm$ 0,5 chez le goupi et $41,0 \pm 0,4 \mathrm{chez}$ le wapa. Ces différences sont statistiquement hautement significatives.

Les courbes pression-volume (fig 4) sont utiles pour déterminer la pression osmotique à saturation (Tyree et Hammel, 1972). Elles montrent que le wapa a un potentiel osmotique $\left(\pi_{0}\right)$ différent de celui du tréma ou du goupi. À partir de l'ensemble des courbes, nous avons en effet des $\pi_{0}$ moyen de 13,0 $\pm 0,6$ bars $(n=4)$ pour le goupi; $13,8 \pm 0,6$ bars $(n=3)$ pour le tréma et de $16,0 \pm 1,7$ bars $(n=3)$ pour le wapa. tives.

Les différences ne sont pas significa-

Les teneurs en eau, à turgescence nulle, déterminées comme étant celles à partir desquelles les courbes pressionvolume deviennent linéaires, sont de $70 \%$ pour tréma, $85 \%$ pour goupi et $90 \%$ pour

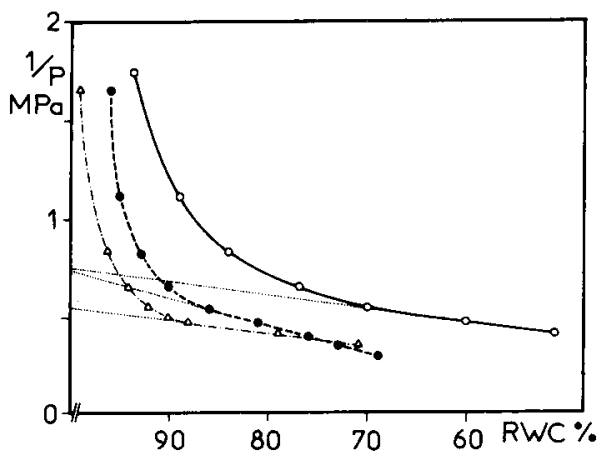

Fig 4. Exemples de courbes pression-volume obtenues à partir de Trema micrantha (- - ), Goupia glabra $(-\mathrm{O}-$ ) et Eperua grandiflora $(-\bullet-\Delta-\cdot-)$. wapa, ce qui va bien dans le sens d'une baisse avec la xérophilie croissante, comme indiqué par Jane et Green (1983). Les effectifs sont cependant trop faibles pour que les différences soient considérées comme significatives.

Le déficit sublétal est un assez bon indice de la résistance cytoplasmique à la diminution de la teneur en eau (Oppenheimer, 1963; Kapos et Tanner, 1985; Rychnovskà-Soudkovà, 1963). L'interprétation des valeurs obtenues reste difficile et parfois subjective. On obtient parfois, au bout de temps de transpiration très longs, une ressaturation, voire une sursaturation rapide des feuilles qui n'a plus rien de physiologique. II importe donc de vérifier la régularité de l'évolution des valeurs en fonction du temps de dessèchement.

Compte tenu de cette remarque, les déficits sublétaux observés au cours des séries de mesures les plus régulières (fig 5) chez nos 3 arbres sont de :

48\% Trema micrantha, 66\% (Goupia glabra) et $88 \%$ (Eperua grandiflora).

II y aurait donc un étalement des valeurs avec une corrélation entre héliophilie et résistance physiologique à la diminution de la teneur en eau.

Rermarquons que le déficit sublétal de tréma, bien qu'inférieur à ceux des autres espèces est atteint beaucoup plus vite par cette plante ( $1 \mathrm{~h}$ environ dans les conditions de l'expérience, c'est-à-dire au soleil) que par les 2 autres espèces (environ $3 \mathrm{~h}$ ).

$\mathrm{Au}$ cours des expériences de déficit sublétal, nous avons effectué quelques observations sur le flétrissement (fanaison). II apparaît une différence marquée en rapport avec la rigidité des feuilles:

- le tréma fane bien avant d'atteindre le déficit sublétal;

- le goupi fane (faiblement) quand il atteint le déficit sublétal;

- le wapa ne fane jamais (feuilles adultes). 


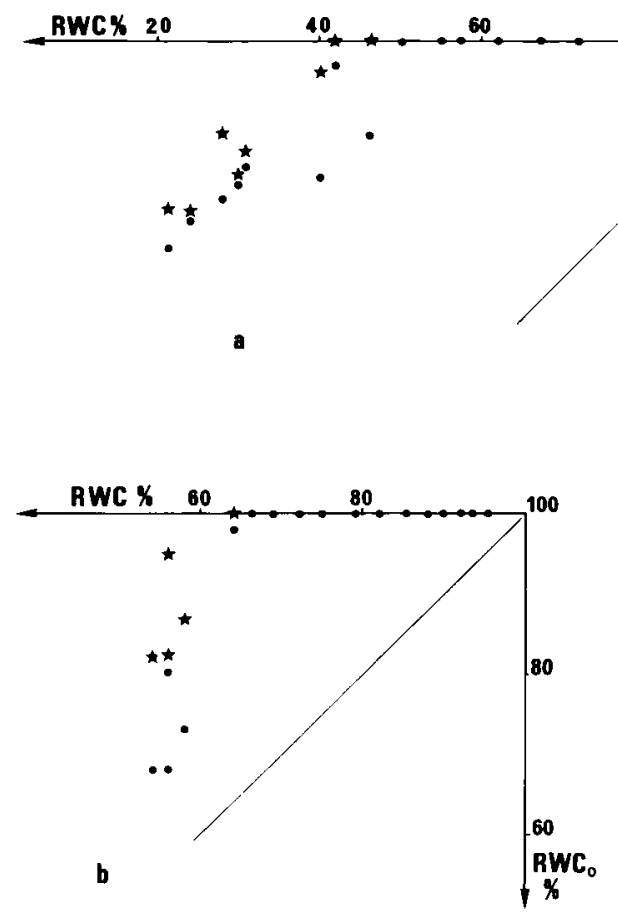

La valeur du déficit sublétal n'est que relative : elle est plus ou moins facilement atteinte par la plante selon son aptitude à puiser l'eau (fonction de son enracinement et de son potentiel), ou à limiter son départ (fonction de la surface foliaire et, surtout, du contrôle stomatique et cuticulaire).

La pesée des rameaux coupés est riche d'informations. La figure 6 illustre le type de réactions observées. Nous avons regroupé dans le tableau I les principales caractéristiques déduites de l'ensemble des courbes : le point de début et de fin de fermeture stomatique exprimé en pourcentage de contenu en eau, le temps pour atteindre la pleine fermeture, la pente de la première et de la deuxième partie droite de la courbe, enfin le temps pour atteindre le déficit sublétal. Les cinétiques de transpiration sont d'ordre 1 : les quantités d'eau transpirées sont proportionnelles aux
Fig 5. Déficit sublétal : réhydratation au bout de $6 \mathrm{~h}(\mathrm{O})$ et $24 \mathrm{~h}(*)$ en fonction du contenu en eau initial : a: Trema micrantha. b: Goupia glabra. c: Eperua grandiflora.

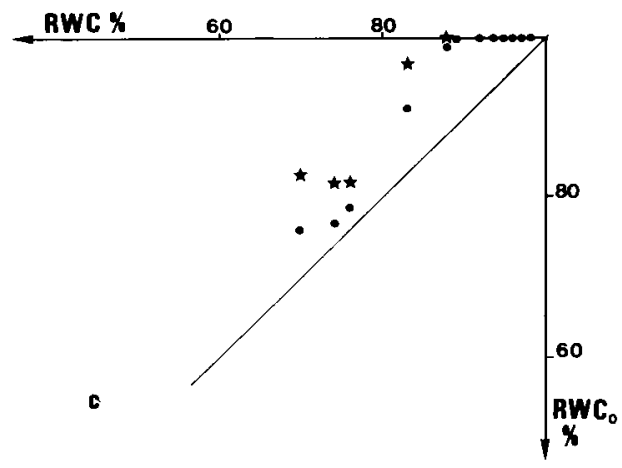

quantités restantes dans les feuilles (Laouar, 1974). La première partie droite correspond à la transpiration stomatique et la deuxième à la transpiration cuticulaire. Sur la figure $6 b$, on observe un cas de non régulation stomatique chez tréma. II s'agit d'un artefact, car le phénomène se produit sur rameau coupé, mais il est intéressant de le signaler, car il ne s'observe que sur des espèces très héliophiles comme les Cecropia, les Inga ou, en Côte d'Ivoire, Terminalia ivorensis. Le fait qu'on l'observe plutôt en air sec que sous fort éclairement semble indiquer que l'absence de régulation stomatique est liée à une déshydratation de l'épiderme foliaire.

On constate que le goupi a très exactement les mêmes caractéristiques que le wapa sauf pour la transpiration cuticulaire, plus faible chez ce dernier. La transpiration très forte du tréma est normale pour une 

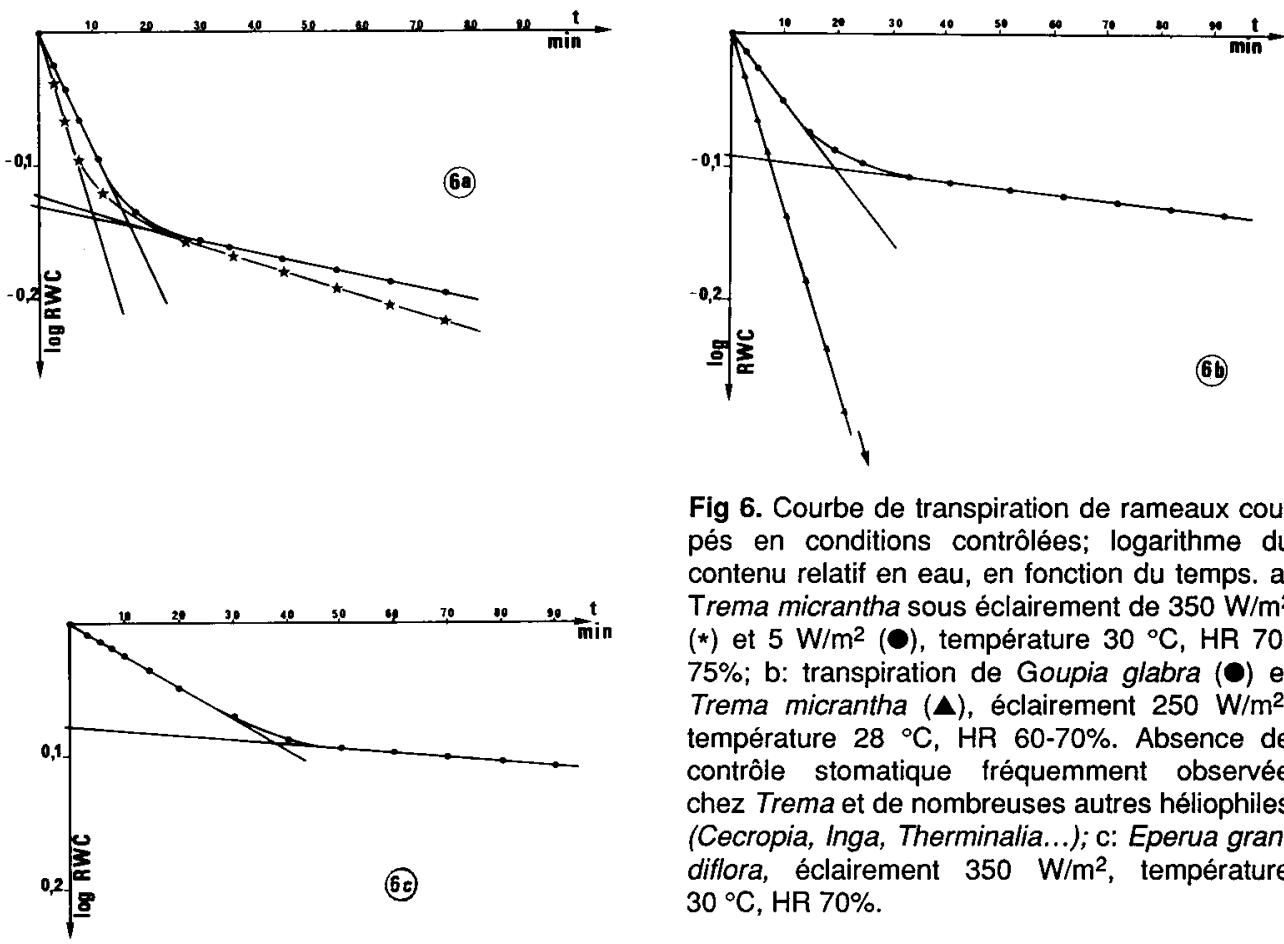

Fig 6. Courbe de transpiration de rameaux coupés en conditions contrôlées; logarithme du contenu relatif en eau, en fonction du temps. a: Trema micrantha sous éclairement de $350 \mathrm{~W} / \mathrm{m}^{2}$ (*) et $5 \mathrm{~W} / \mathrm{m}^{2}(0)$, température $30^{\circ} \mathrm{C}, \mathrm{HR} 70$ $75 \%$; b: transpiration de Goupia glabra (O) et Trema micrantha (A), éclairement $250 \mathrm{~W} / \mathrm{m}^{2}$, température $28{ }^{\circ} \mathrm{C}$, HR $60-70 \%$. Absence de contrôle stomatique fréquemment observée chez Trema et de nombreuses autres héliophiles (Cecropia, Inga, Therminalia...); c: Eperua grandiflora, éclairement $350 \mathrm{~W} / \mathrm{m}^{2}$, température $30^{\circ} \mathrm{C}, \mathrm{HR} 70 \%$.

espèce à croissance très rapide. Tréma transpire plus que le goupi ou le wapa et malgré une régulation stomatique précoce dans le temps, il atteint rapidement des valeurs très basses de teneur en eau. Sa régulation transpiratoire liée à la surface

foliaire totale s'ajuste, grâce à un renouvellement très rapide des feuilles; nous avons ainsi estimé l'indice foliaire à 2 en saison humide et moins de 1 en saison sèche. Le goupi et le wapa jeunes ne présentent pas de réduction sensible de la surface foliaire.

Tableau I. Caractéristiques de la tranpiration par la technique de pesée des rameaux coupés : turgescence relative en début et fin de fermeture stomatique; conductances stomatiques $\left(a_{1}\right)$ et cuticulaires $\left(a_{2}\right)$ (pentes des parties droites des courbes de transpiration, unités arbitraires); temps pour atteindre la teneur en eau correspondant au déficit sublétal. Les rameaux coupés sont placés à l'ombre dans des conditions d'évaporation modérée.

\begin{tabular}{lcccccc}
\hline & $\begin{array}{c}\text { Début } \\
\text { fermeture } \\
(\%)\end{array}$ & $\begin{array}{c}\text { Fin } \\
\text { fermeture } \\
(\%)\end{array}$ & $\begin{array}{c}\text { Temps } \\
\text { fermeture } \\
(\text { min) }\end{array}$ & $a_{1}$ & $a_{2}$ & $\begin{array}{c}\text { Temps } \\
D S L(h)\end{array}$ \\
\hline Trema & $79 \pm 2$ & $67 \pm 4$ & $29 \pm 6$ & $67 \pm 5$ & $10 \pm 5$ & 2 \\
Goupi & $87 \pm 2$ & $77 \pm 8$ & $40 \pm 10$ & $45 \pm 10$ & $10 \pm 3$ & 6 \\
Wapa & $83 \pm 3$ & $75 \pm 5$ & $40 \pm 10$ & $45 \pm 15$ & $5 \pm 3$ & 6 \\
\hline
\end{tabular}


La fanaison des feuilles de tréma est comme une régulation supplémentaire, en réduisant l'apport de lumière (Chiariello et al, 1987).

La figure 7 représente le système racinaire des 3 espèces. Les différences morphologiques sont considérables. Tréma possède un système de racines extrêmement simple : 1 disque ou plateau de racines à la surface du sol et un court pivot qui se prolonge, vraisemblablement très profondément, par des racines fines. Chez le goupi, les racines horizontales sont également les plus développées, mais elles ne forment pas un disque comme chez le tréma et sont moins superficielles (Alexandre, 1988). II n'y a pas de pivot proprement dit, mais un grand nombre d'axes rayonnants; la profondeur atteinte par ces axes obliques est faible chez la jeune plante. Le goupi présente souvent des feuilles petites, minces et claires, parfois rougeâtres. L'hypothèse d'une nématose a pu être écartée après test avec un némati- cide et observation directe de Scotto la Massese (INRA, Antibes, communication personnelle). Un apport de fertilisants du commerce n'a pas eu d'effets. Nous pensons que la plante peut présenter une difficulté de micorhization qui accroît les risques de déficit hydrique. Les quelques observations effectuées sur le wapa montrent un système racinaire composé d'assez grosses racines peu nombreuses mais profondes.

\section{DISCUSSION}

Le fait principal qui ressort de cette série de mesures, c'est la grande différence de comportement entre espèces. Ces différences sont aggravées par la sécheresse, même si celle-ci est peu accentuée. En fait, la sécheresse de 1987 est particulièrement prononcée, mais pas exceptionnelle. À Barro Colorado, sous un climat assez proche du climat guyanais, Fetcher (1979)

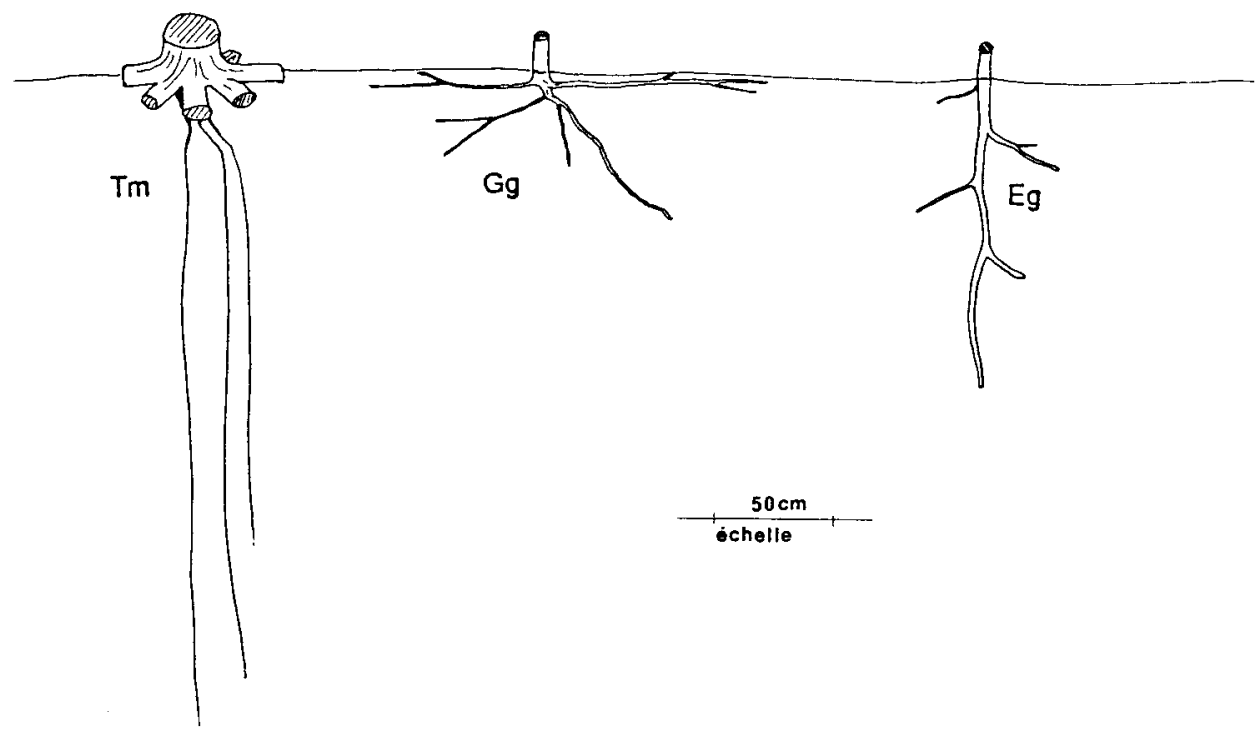

Fig 7. Dessin schématique de l'appareil racinaire, à 3 ans, de Trema micrantha (Tm), Goupia glabra $(\mathrm{Gg})$ et Eperua grandiflora $(\mathrm{Eg})$. 
arrive à une conclusion identique. II montre par exemple que le potentiel de base de Trichilia cipo passe de -26 bars en fin de saison sèche à -4 dès les premières pluies. La baisse du potentiel de base s'accompagne d'une baisse de la conductance stomatique.

Les espèces spontanées, avec l'exemple de tréma, donc à leur place dans la succession végétale, souffrent bien moins que celles qui sont normalement plus tardives, ici goupi et surtout wapa. Les espèces pionnières seraient ainsi plus résistantes à la sécheresse que les espèces forestières. Ceci va dans le sens de l'existence d'une nécessaire succession végétale au sens de Clements. Il y aurait facilitation (au sens de Connell et Slatyer) dans la mesure où la végétation pionnière contribue à créer une "ambiance forestière" plus humide que le macroclimat. En fait la notion de résistance à la sécheresse est bien complexe. II faut notamment éviter de couper la plante de ses racines. En effet une plante peut se montrer sensible par la physiologie de ses feuilles mais en fait résistante in situ grâce à ses racines (Aussenac et al, 1989). Ici les différences de morphologie racinaire rencontrées sont importantes et il n'y a pas de doute qu'elles peuvent avoir une large répercussion dans l'alimentation hydrique en période sèche. C'est un point qui reste à approfondir en priorité.

On peut parler de résistance à la sécheresse dans les mêmes termes que ceux utilisés pour la résistance aux maladies. II existe alors 3 types de résistance au sens large : l'immunité où la plante ignore le manque d'eau en ayant une absorption suffisante et éventuellement des réserves, la résistance proprement dite où la plante limite ses pertes, enfin la tolérance où la plante fonctionne encore malgré un bilan hydrique déficitaire.
Aucune de nos espèces n'est véritablement résistante à la sécheresse et n'approche des caractéristiques observées par Okali (1971) dans la plaine d'Accra au Ghana, avec notamment des teneurs en eau critiques de l'ordre de $30 \%$. Si l'on s'autorise néanmoins à parler de résistance à la sécheresse chez nos 3 arbres, chacun d'eux illustre 1 des 3 tendances fondamentales. Tréma est immun grâce surtout à ses racines profondes, mais également grâce à la forte teneur en eau de ses tissus. En conservant une transpiration élevée, tréma peut continuer à croître malgré la sécheresse relative et peut ainsi conserver dans les recrûs sa position dominante, ce qui est nécessaire à sa survie car c'est une espèce strictement héliophile. Cette stratégie écophysiologique le met en revanche à la merci d'une sécheresse trop accentuée. La stratégie de tréma semble caractéristique de très nombreuses espèces pionnières. C'est un peu la politique du tout ou rien. La conductance stomatique maximale qui dépasse $2 \mathrm{~cm} / \mathrm{s}$ est particulièrement élevée, mais bien en relation avec la rapidité de croissance (Korner et al, 1979).

Le goupi, lui, est plutôt tolérant; en effet il continue à transpirer malgré un bilan déficitaire et le wapa est plutôt résistant avec son épaisse cuticule qui se retrouve chez la grande majorité des espèces les plus "forestières". Ce caractère bien connu (Richards, 1952) ne semble guère avoir d'utilité sur le plan hydrique (Kapos et Tanner, 1985). On peut être tenté de lui chercher une autre utilité, comme celle de conserver le $\mathrm{CO}_{2}$ respiratoire ou d'offrir une protection à l'encontre des maladies. L'épaisseur de la cuticule pourrait aussi n'être que la conséquence d'un déficit dans l'alimentation minérale (Peace et MacDonald, 1981). On notera toutefois que d'autres études font état d'une plus grande résistance à la sécheresse d'espèces forestières compa- 
rées à des espèces secondaires. Ainsi en Guyane, selon Huc et Guehl (1989), un autre wapa (E falcata) montre des caractéristiques de résistance supérieures à celles de l'espèce de recrû Jacaranda copaia. À Barro Collorado, les mesures de Rundel (Robichaux et al, 1984) montrent que les forestières, dont Trichilia tuberculata, sont plus résistantes à la sécheresse que des secondaires comme Cordia alliodora. En milieu subtropical montagnard, Jane et Green (1983) arrive à cette même conclusion. Si l'on considère le faible déficit hydrique qui amène à la perte de turgescence, caractère qui se retrouve chez d'autres arbres forestiers comme Pentaclethra macroloba (Oberbauer, 1982), les caractères de résistance deviennent simplement corrélatifs de caractères de sensibilité. Par contre, si on replace nos 3 espèces dans la stratégie évolutive où, comme l'indiquent Vartanian et Lemée (1984), le dilemne de base est survivre ou produire, il apparaît bien que la tactique axée sur la survie est en rapport avec une stratégie plus générale de type «K» tandis que la "gambling strategy" des espèces de type "r" ira de pair avec une croissance maximalisée au prix d'un risque accrû pour la survie. La stratégie de tolérance du goupi, plus primitive selon Vartanian et Lemée (1984), est quant à elle, bien en rapport avec l'écologie de cette plante qui se montre extrêmement souple. Nulle part dominante, elle se comporte très bien aussi bien à l'ombre qu'en pleine lumière (Alencar et Araujo, 1980; Lacoste, 1990).

\section{REMERCIEMENTS}

L'auteur tient à remercier $\mathrm{P}$ Cruiziat pour ses conseils dans l'élaboration du protocole expérimental, JY Pontailler pour la réalisation des figures et les 2 lecteurs anonymes pour la révision du manuscrit.

\section{RÉFÉRENCES}

Alencar JC, Araujo VC (1980) Comportamento de especies florestais amazonicas quanto à luminosidade. Acta Amazonica 10, 435-444

Alexandre DY (1988) Morphologie racinaire en relation avec l'organisation du sol : cas de deux espèces pionnières de Guyane française : Goupia glabra et Vismia guianensis. Cah ORSTOM Sér Pédol (à paraître)

Alexandre DY (1989) Pluies et alimentation en eau des plantes dans la région de Sinnamary (Guyane française). Nature guyanaise 3, 2836

Aussenac G (1985) Le potentiel hydrique de l'arbre : une donnée essentielle pour la compréhension de l'écophysiologie des essences forestières. Sci Sol 4, 217-226

Aussenac $G$ et al (1989) Drought resistance of two Douglas fir species (Pseudotsuga menziesii and $P$ macrocarpa): relative importance of water use efficiency and root growth potential. In: Forest tree physiology (Dreyer et al, eds) Elsevier INRA, 384-387

Bannister $P$ (1971) The water relation of heathplants from open and shade habitats. $J E c o l$ $59,51-64$

Chiariello NR et al (1987) Midday wilting in a tropical pioneer tree. Func Ecol 1, 3-11

Colin F (1989) Ecophysiologie en forêt équatoriale. Contribution à la connaissance de Goupia glabra. Rapport de stage ENGREF Kourou $37 \mathrm{p}$

Fetcher $N$ (1979) Water relations of five tropical tree species on Barro Colorado Island, Panama. CEcologia (Berl) 40, 229-233

Forget PM (1988) Dissémination et régénération naturelle de huit espèces d'arbres en forêt guyanaise. Thèse Paris $6,232 p$

Hallé F, Oldeman RAA (1970) Essai sur l'architecture et la dynamique de croissance des arbres tropicaux. Paris, Masson, $178 p$

Huc R, Guehl JM (1989) Environmental control of $\mathrm{CO}_{2}$ assimilation rate and leaf conductance in two species of the tropical rain forest of French Guiana (Jacaranda copaia D Dom and Eperua falcata Aubl). Multigr INRA $3 p$

Jane GT, Green TGA (1983) Utilisation of pressure-volume techniques and non linear least 
squares analysis to investigate site induced stress in evergreen trees. CEcologia (Berl) 57, 380-390

Kapos V, Tanner EVJ (1985) Water relations of Jamaican upper montane rain forest trees. Ecology 66, 241-250

Korner $C$ et al (1979) Maximum leaf diffusive conductance in vascular plants. Photosynthetica (Prague) 13, 45-82

Lacoste JF (1990) Effet de la suppression d'arbres pionniers sur l'évolution d'un jeune recrû forestier guyanais. Thèse Orsay, $81 p$

Laouar $S$ (1974) Variation de la résistance foliaire à la diffusion de vapeur d'eau au cours du dessèchement de la feuille. Bull Ecol 12, $11-112$

Oberbauer SF (1982) Water relations of Pentaclethra macroloba a wet tropical forest tree. Bull Ecol Soc Am 63, 178

Okali DUU (1971) Tissue water relations of some woody species of the Accra plains, Ghana. J Ecol 59, 89-101

Oppenheimer HR (1963) Zur Kenntnis kritischer Wasser sattigungsdefizite in Blattern und ihrer Bestimmung. Planta 60, 51-69
Peace WJ, MacDonald FD (1981) An investigation of the leaf anatomy, foliar mineral levels, and water relations of trees of a Sarawak forest. Biotropica 13, 100-109

Reich PB, Borchert R (1988) Changes with leaf age in the stomatal function and water status of several tropical tree species. Biotropica 20, 60-69

Richards PW (1952) The tropical rain forest. Cambridge University Press, $450 \mathrm{p}$

Robichaux RH et al (1984) Tissue water deficit and plant growth in wet tropical environments. In: Physiological ecology of plants of the wet tropics. (Medina E, Mooney HA, Vazquez-Yanes $C$, eds) W Junk, 99-112

Rychnovská-Soudková M (1963) Study of the reversibility of the water saturation deficit as one of the methods of causal phytogeography. Biol Plant (Prague) 5, 175-180

Tyree MT, Hammel HT (1972) The measurement of the turgor pressure and the water relations of plants by the pressure bomb technique. $J$ Exp Bot 23, 267-282

Vartanian N, Lemée G (1984) La notion d'adaptation à la sécheresse. Bull Soc Bot Fr, ACtual Bot 131, 7-15 